\title{
STABILITY ANALYSIS OF HIGHLY NONLINEAR HYBRID MULTIPLE-DELAY STOCHASTIC DIFFERENTIAL EQUATIONS*
}

\author{
Chen $\mathrm{Fei}^{1}$, Weiyin $\mathrm{Fei}^{2, \dagger}$, Xuerong $\mathrm{Mao}^{3}$, Mingxuan Shen ${ }^{2}$ and Litan \\ $\operatorname{Yan}^{1}$
}

\begin{abstract}
Stability criteria for stochastic differential delay equation (SDDE) have been studied intensively for the past few decades. However, most of these criteria can only be applied to delay equations where their coefficients are either linear or nonlinear but bounded by linear functions. Recently, the stability of highly nonlinear hybrid stochastic differential equations with a single delay is investigated in [Fei, Hu, Mao and Shen, Automatica, 2017], whose work, in this paper, is extended to highly nonlinear hybrid stochastic differential equations with variable multiple delays. In other words, this paper establishes the stability criteria of highly nonlinear hybrid variable multipledelay stochastic differential equations. We also discuss an example to illustrate our results.
\end{abstract}

Keywords Variable multiple-delay stochastic differential equation; nonlinear growth condition; asymptotic stability; Markovian switching; Lyapunov functional.

MSC(2010) 60H10, 34K20, 93E15.

\section{Introduction}

In many practical systems, such as science, industry, economics and finance etc., we will encounter the systems with time delay. Differential delay equations (DDEs) have been employed to model such time-delay systems. Since the time-delay often causes the instability of systems, stability of DDEs has been explored intensively for more than 50 years. Generally, the stability criteria are classified into the delayindependent and delay-dependent stability criteria. When the size of delays of the systems is incorporated into the delay-dependent stability criteria, the delaydependent systems are generally less conservative than the delay-independent ones which work for any size of delays. There exists a very rich literature in this topics

${ }^{\dagger}$ The corresponding author. Email address: wyfei@ahpu.edu.cn(W. Fei)

${ }^{1}$ Glorious Sun School of Business and Management, Donghua University, Shanghai, 200051, China

${ }^{2}$ School of Mathematics and Physics, Anhui Polytechnic University, Wuhu, Anhui, 241000, China

${ }^{3}$ Department of Mathematics and Statistics, University of Strathclyde, Glasgow, G1 1XH, U.K.

*The authors would like to thank Liangjian $\mathrm{Hu}$ and Wei Liu for their valuable comments and suggestions. This paper is supported by the Natural Science Foundation of China (71571001) for the financial support. 
(see, e.g., $[3,11-13,16,17,21,36])$.

Since 1980's, stochastic differential delay equations were investigated in order to model practical systems which are subject to external noises (see, e.g., [27]). Since then, the study of the stability on SDDEs has been one of the most important topics (see, e.g., $[5,10,15,19,20,24]$ ).

In 1990's, hybrid SDDEs (called also SDDEs with Markovian switching) were developed to model real-world systems since they may experience abrupt changes in their parameters and structure in addition to uncertainties and time lags. One of the important issues in the research of hybrid SDDEs is the analysis of stability of control systems. Moreover the delay-dependent stability criteria have been erected by many authors (see, e.g., [2, 4, 22, 23, 25, 26, 28,33-35]). To our best knowledge, the existing delay-dependent stability criteria are mainly created for the hybrid SDDEs where their coefficients are either linear or nonlinear but bounded by linear functions. Based on highly nonlinear hybrid SDDEs (see, e.g., [6-9,14,15,30-32]), [7] has recently established the delay-dependent stability criterion where they solve the stability of a single delay system. However, many real systems has multiple timedelay states (see, e.g., $[1,18,29])$. Therefore we further develop the stability criteria of highly nonlinear hybrid SDDEs with variable multiple delays.

Specifically, we first discuss the following SDDE with two delays $\delta_{1}(t), \delta_{2}$ with $\delta_{1}(t) \leq \tau$ (see Example 4.1)

$$
d x(t)=\left\{\begin{array}{l}
\left(-10 x^{3}(t)-x\left(t-\delta_{1}(t)\right)\right) d t+\frac{1}{4} x^{2}\left(t-\delta_{2}(t)\right) d B(t), \text { if } r(t)=1, \\
\left(-4 x^{3}(t)+\frac{1}{2} x\left(t-\delta_{1}(t)\right)\right) d t+\frac{1}{2} x^{2}\left(t-\delta_{2}(t)\right) d B(t), \text { if } r(t)=2,
\end{array}\right.
$$

on $t \geq 0$ with initial data

$$
\{x(u)=2+\sin (u):-\tau \leq u \leq 0\} \in C([-\tau, 0] ; \mathbb{R}), r(0)=i_{0} \in \mathbb{S} .
$$

Here $B(t)$ is a scalar Brownian motion, $r(t)$ is a Markovian chain with space $\mathbb{S}=$ $\{1,2\}$ and its generator $\Gamma$ given by

$$
\Gamma=\left(\begin{array}{cc}
-1 & 1 \\
8 & -8
\end{array}\right)
$$

The above system (1.1) will switch from one mode to the other according to the probability law of the Markovian chain. If $\delta_{1}(t) \leq \tau=0.01$, the computer simulation shows it is asymptotically stable (see Figure 4.1 ). If the time-delay is large, say $\delta_{1}(t) \leq \tau=2$, the computer simulation shows that the hybrid multiple-delays SDE (1.1) is unstable (see Figure 4.2 ). In other words, whether the hybrid multipledelay SDE is stable or not depends on how small or large the time-delay is. On the other hand, both drift and diffusion coefficients of the hybrid SDE with multiple delays affect the stability of systems due to highly nonlinear. However, there is no delay dependent criterion which can be applied to the SDE with multiple delays to derive a sufficient bound on the time-delay $\tau$ such that the SDDE is stable, although the stability criteria of the highly nonlinear hybrid SDE with single delay have been created in [7]. This paper first established delay dependent criteria for highly nonlinear hybrid SDEs with variable multiple delays.

In comparison with [7], the key contributions in this paper are highlighted below: 
- This paper takes the variable multiple delays into account to develop a new theory on the robust stability and boundedness for highly nonlinear hybrid SDDEs.

- The new theory established in this paper is applicable to hybrid SDDEs with different delays in drift and diffusion coefficient of SDDEs with multiple delays (see (2.1)). Especially, we found that the sizes of delays in drift coefficient only affect the stability of the system, but the sizes of delays in the diffusion coefficient do NOT. This result has a significant importance.

- A significant amount of new mathematics has been developed to deal with the difficulties due to different delays in drift and diffusion coefficient of SDDEs with multiple delays and those without the linear growth condition. For example, a more complicated Lyapunov function will be designed in order to deal with the effects of the different delays. A lot of effort has also been put into showing the bounds of the sizes of delays.

To develop our new theory, we will introduce some necessary notation in Section 2. We will discuss in Section 3 the delay-dependent asymptotic stability of SDEs with variable multiple delays, and give main results on robust boundedness and stability. We will present an example in Section 4 to illustrate our theory. We will finally conclude our paper in Section 5.

\section{Notation and Assumptions}

Throughout this paper, unless otherwise specified, we use the following notation. If $A$ is a vector or matrix, its transpose is denoted by $A^{\top}$. If $x \in \mathbb{R}^{d}$, then $|x|$ is its Euclidean norm. For a matrix $A$, we let $|A|=\sqrt{\operatorname{trace}\left(A^{\top} A\right)}$ be its trace norm and $\|A\|=\max \{|A x|:|x|=1\}$ be the operator norm. Let $\mathbb{R}_{+}=[0, \infty)$. For $\tau>0$, denote by $C\left([-\tau, 0] ; \mathbb{R}^{d}\right)$ the family of continuous functions $\eta$ from $[-\tau, 0] \rightarrow \mathbb{R}^{d}$ with the norm $\|\eta\|=\sup _{-\tau<u<0}|\eta(u)|$. If $A$ is a subset of $\Omega$, denote by $I_{A}$ its indicator function. Let $\left(\Omega, \mathcal{F},\left\{\mathcal{F}_{t}\right\}_{t>0}, \mathbb{P}\right)$ be a complete probability space with a filtration $\left\{\mathcal{F}_{t}\right\}_{t \geq 0}$ satisfying the usual conditions. Let $B(t)=\left(B_{1}(t), \cdots, B_{m}(t)\right)^{\top}$ be an $m$-dimensional Brownian motion defined on the probability space. Let $r(t)$, $t \geq 0$, be a right-continuous Markov chain on the probability space taking values in a finite state space $\mathbb{S}=\{1,2, \cdots, N\}$ with generator $\Gamma=\left(\gamma_{i j}\right)_{N \times N}$ given by

$$
\mathbb{P}\{r(t+\Delta)=j \mid r(t)=i\}= \begin{cases}\gamma_{i j} \Delta+o(\Delta) & \text { if } i \neq j, \\ 1+\gamma_{i i} \Delta+o(\Delta) & \text { if } i=j,\end{cases}
$$

where $\Delta>0$. Here $\gamma_{i j} \geq 0$ is the transition rate from $i$ to $j$ if $i \neq j$ while $\gamma_{i i}=-\sum_{j \neq i} \gamma_{i j}$. We assume that the Markov chain $r(\cdot)$ is independent of the Brownian motion $B(\cdot)$. Let $\tau_{j}, \bar{\delta}_{j} \in[0,1), j=1, \cdots, n$, be constants with $\tau=$ : $\max _{j=1}^{n_{1}} \tau_{j}$. The delays $\delta_{j}(\cdot)$ are differential functions from $\mathbb{R}_{+} \rightarrow[0, \tau]$, such that $\dot{\delta}_{j}(t):=d \delta_{j}(t) / d t \leq \bar{\delta}_{j}$ for all $t \leq 0$, and $\tau_{j} \geq \delta_{j}(t)$. For Borel measurable functions

$$
f: \mathbb{R}^{d\left(n_{1}+1\right)} \times \mathbb{S} \times \mathbb{R}_{+} \rightarrow \mathbb{R}^{d} \text { and } g: \mathbb{R}^{d\left(n-n_{1}+1\right)} \times \mathbb{S} \times \mathbb{R}_{+} \rightarrow \mathbb{R}^{d \times m},
$$

we consider a $d$-dimensional hybrid SDE with $n$-delays

$$
d x(t)=f\left(x(t), x\left(t-\delta_{1}(t)\right), \cdots, x\left(t-\delta_{n_{1}}(t)\right), r(t), t\right) d t
$$




$$
+g\left(x(t), x\left(t-\delta_{n_{1}+1}(t)\right), \cdots, x\left(t-\delta_{n}(t)\right), r(t), t\right) d B(t)
$$

on $t \geq 0$ with initial data

$$
\{x(t):-\tau \leq t \leq 0\}=\eta \in C\left([-\tau, 0] ; \mathbb{R}^{d}\right), r(0)=i_{0} \in \mathbb{S}
$$

The classical conditions for the existence and uniqueness of the global solution are the local Lipschitz condition and the linear growth condition (see, e.g., [24]). In this paper, we need only the local Lipschitz condition. However, we will consider highly nonlinear hybrid SDEs with multiple delays which, in general, do not satisfy the linear growth condition in this paper. Therefore, we impose the polynomial growth condition, instead of the linear growth condition. Let us state these conditions as an assumption for our aim.

Assumption 2.1. Assume that for any $h>0$, there exists a positive constant $K_{h}$ such that

$$
\begin{aligned}
& \left|f\left(x, y_{1}, \cdots, y_{n_{1}}, i, t\right)-f\left(\bar{x}, \bar{y}_{1}, \cdots, \bar{y}_{n_{1}}, i, t\right)\right| \\
& \vee\left|g\left(x, y_{n_{1}+1}, \cdots, y_{n}, i, t\right)-g\left(\bar{x}, \bar{y}_{n_{1}+1}, \cdots, \bar{y}_{n}, i, t\right)\right| \\
& \quad \leq K_{h}\left(|x-\bar{x}|+\sum_{j=1}^{n}\left|y_{j}-\bar{y}_{j}\right|\right)
\end{aligned}
$$

for all $x, y_{1}, \cdots, y_{n}, \bar{x}, \bar{y}_{1}, \cdots, \bar{y}_{n} \in \mathbb{R}^{d}$ with $|x| \vee\left|y_{1}\right| \vee \cdots \vee\left|y_{n}\right| \vee \cdots \vee|\bar{x}| \vee\left|\bar{y}_{1}\right| \vee \cdots \vee$ $\left|\bar{y}_{n}\right| \leq h$ and all $(i, t) \in \mathbb{S} \times \mathbb{R}_{+}$. Assume moreover that there exist three constants $K>0, q_{1} \geq 1$ and $q_{2} \geq 1$ such that

$$
\begin{aligned}
& \left|f\left(x, y_{1}, \cdots, y_{n_{1}}, i, t\right)\right| \leq K\left(1+|x|^{q_{1}}+\sum_{j=1}^{n_{1}}\left|y_{j}\right|^{q_{1}}\right), \\
& \left|g\left(x, y_{n_{1}+1}, \cdots, y_{n}, i, t\right)\right| \leq K\left(1+|x|^{q_{2}}+\sum_{j=n_{1}+1}^{n}\left|y_{j}\right|^{q_{2}}\right)
\end{aligned}
$$

for all $x, y_{1}, \cdots, y_{n} \in \mathbb{R}^{d},(i, t) \in \mathbb{S} \times \mathbb{R}_{+}$.

If $q_{1}=q_{2}=1$, then condition (2.3) is the familiar linear growth condition. However, we emphasise once again that we are here interested in highly nonlinear multiple-delay SDEs which have either $q_{1}>1$ or $q_{2}>1$. We will refer condition (2.3) as the polynomial growth condition. It is known that Assumption 2.1 only guarantees that the $\operatorname{SDDE}(2.1)$ with the initial data (2.2) has a unique maximal solution, which may explode to infinity at a finite time. To avoid such a possible explosion, we need to impose an additional condition in terms of Lyapunov functions. For this purpose, we need more notation.

Let $C^{2,1}\left(\mathbb{R}^{d} \times \mathbb{S} \times \mathbb{R}_{+} ; \mathbb{R}_{+}\right)$denote the family of non-negative functions $U(x, i, t)$ defined on $(x, i, t) \in \mathbb{R}^{d} \times \mathbb{S} \times \mathbb{R}_{+}$which are continuously twice differentiable in $x$ and once in $t$. For such a function $U(x, i, t)$, let $U_{t}=\frac{\partial U}{\partial t}, U_{x}=\left(\frac{\partial U}{\partial x_{1}}, \cdots, \frac{\partial U}{\partial x_{d}}\right)$, and $U_{x x}=\left(\frac{\partial^{2} U}{\partial x_{k} \partial x_{l}}\right)_{d \times d}$. Let $C\left(\mathbb{R}^{d} \times[-\tau, \infty) ; \mathbb{R}_{+}\right)$denote the family of all continuous functions from $\mathbb{R}^{d} \times[-\tau, \infty)$ to $\mathbb{R}_{+}$. We can now state another assumption. 
Assumption 2.2. Assume that there exists a pair of functions $\bar{U} \in C^{2,1}\left(\mathbb{R}^{d} \times \mathbb{S} \times\right.$ $\left.\mathbb{R}_{+} ; \mathbb{R}_{+}\right)$and $G \in C\left(\mathbb{R}^{d} \times[-\tau, \infty) ; \mathbb{R}_{+}\right)$, as well as positive numbers $c_{1}, c_{2}, c_{3, j}$ and $q \geq 2\left(q_{1} \vee q_{2}\right)$, such that

$$
\sum_{j=1}^{n} \frac{c_{3, j}}{1-\bar{\delta}_{j}}<c_{2}, \quad|x|^{q} \leq \bar{U}(x, i, t) \leq G(x, t)
$$

for $\forall(x, i, t) \in \mathbb{R}^{d} \times \mathbb{S} \times \mathbb{R}_{+}$,

and

$$
\begin{aligned}
& \mathbb{L} \bar{U}\left(x, y_{1}, \cdots, y_{n}, i, t\right):=\bar{U}_{t}(x, i, t)+\bar{U}_{x}(x, i, t) f\left(x, y_{1}, \cdots, y_{n_{1}}, i, t\right) \\
& +\frac{1}{2} \operatorname{trace}\left[g^{T}\left(x, y_{n_{1}+1}, \cdots, y_{n}, i, t\right) \bar{U}_{x x}(x, i, t) g\left(x, y_{n_{1}+1}, \cdots, \cdots, y_{n}, i, t\right)\right] \\
& +\sum_{j=1}^{N} \gamma_{i j} \bar{U}(x, j, t) \\
& \leq c_{1}-c_{2} G(x, t)+\sum_{j=1}^{n} c_{3, j} G\left(y_{j}, t-\delta_{j}(t)\right)
\end{aligned}
$$

for all $x, y_{1}, \cdots, y_{n} \in \mathbb{R}^{d},(i, t) \in \mathbb{S} \times \mathbb{R}_{+}$.

Similar to the discussion in [14], we have the following claim.

Lemma 2.1. Under Assumptions 2.1 and 2.2, the variable multiple-delay SDE (2.1) with the initial data (2.2) has the unique global solution $x(t)$ on $t \geq-\tau$ and the solution has the property that

$$
\sup _{-\tau \leq t<\infty} \mathbb{E}|x(t)|^{q}<\infty .
$$

\section{Delay-Dependent Asymptotic Stability of SDEs with Variable Multiple Delays}

In Lemma 2.1, we used the method of Lyapunov functions to study the existence and uniqueness of the solution of the highly nonlinear hybrid SDE (2.1). In this section, we will use the method of Lyapunov functionals to investigate the delay-dependent asymptotic stability. We define two segments $\bar{x}_{t}:=\{x(t+s):-2 \tau \leq s \leq 0\}$ and $\bar{r}_{t}:=\{r(t+s):-2 \tau \leq s \leq 0\}$ for $t \geq 0$. For $\bar{x}_{t}$ and $\bar{r}_{t}$ to be well defined for $0 \leq t<2 \tau$, we set $x(s)=\eta(-\tau)$ for $s \in[-2 \tau,-\tau)$ and $r(s)=r_{0}$ for $s \in[-2 \tau, 0)$. We construct the Lyapunov functional as follows

$$
\begin{aligned}
V\left(\bar{x}_{t}, \bar{r}_{t}, t\right) & =U(x(t), r(t), t) \\
+ & \sum_{j=1}^{n_{1}} \theta_{j} \int_{-\tau_{j}}^{0} \int_{t+s}^{t}\left[\tau_{j}\left|f\left(x(v), x\left(v-\delta_{1}(v)\right), \cdots, x\left(v-\delta_{n_{1}}(v)\right), r(v), v\right)\right|^{2}\right. \\
+ & \left.\left|g\left(x(v), x\left(v-\delta_{n_{1}+1}(v)\right), \cdots, x\left(v-\delta_{n}(v)\right), r(v), v\right)\right|^{2}\right] d v d s
\end{aligned}
$$

for $t \geq 0$, where $U \in C^{2,1}\left(\mathbb{R}^{d} \times \mathbb{S} \times \mathbb{R}_{+} ; \mathbb{R}_{+}\right)$such that

$$
\lim _{|x| \rightarrow \infty}\left[\inf _{(t, i) \in \mathbb{R}_{+} \times \mathbb{S}} U(x, t, i)\right]=\infty
$$


and $\theta_{j}, j=1, \cdots, n$ are positive numbers to be determined later while we set

$$
\begin{aligned}
& f\left(x, y_{1}, \cdots, y_{n_{1}}, i, s\right)=f\left(x, y_{1}, \cdots, y_{n_{1}}, i, 0\right), \\
& g\left(x, y_{n_{1}+1}, \cdots, y_{n}, i, s\right)=g\left(x, y_{n_{1}+1}, \cdots, y_{n}, i, 0\right)
\end{aligned}
$$

for all $x, y_{1}, \cdots, y_{n} \in \mathbb{R}^{d},(i, s) \in \mathbb{S} \times[-2 \tau, 0)$. Applying the generalized Itô formula (see, e.g., [26, Theorem 1.45 on page 48]) to $U(x(t), r(t), t)$, we get

$$
\begin{aligned}
& d U(x(t), r(t), t)=\left(U_{t}(x(t), r(t), t)\right. \\
& +U_{x}(x(t), r(t), t) f\left(x(t), x\left(t-\delta_{1}(t)\right), \cdots, x\left(t-\delta_{n_{1}}(t)\right), r(t), t\right) \\
& +\frac{1}{2} \operatorname{trace}\left[g^{T}\left(x(t), x\left(t-\delta_{n_{1}+1}(t)\right), \cdots, x\left(t-\delta_{n}(t)\right), r(t), t\right)\right. \\
& \left.\quad \times U_{x x}(x(t), r(t), t) g\left(x(t), x\left(t-\delta_{n_{1}+1}(t)\right), \cdots, x\left(t-\delta_{n}(t)\right), r(t), t\right)\right] \\
& \left.+\sum_{j=1}^{N} \gamma_{r(t), j} U(x(t), j, t)\right) d t+d M(t),
\end{aligned}
$$

for $t \geq 0$, where $M(t)$ (see, e.g., [26, Theorem 1.45 on page 48]) is a continuous local martingale with $M(0)=0$. Rearranging terms gives

$$
\begin{aligned}
d U & (x(t), r(t), t) \\
\quad & \left(U _ { x } ( x ( t ) , r ( t ) , t ) \left[f\left(x(t), x\left(t-\delta_{1}(t)\right), \cdots, x\left(t-\delta_{n_{1}}(t)\right), r(t), t\right)\right.\right. \\
& \quad-f(x(t), x(t), \cdots, x(t), r(t), t)] \\
& \left.+\mathcal{L} U\left(x(t), x\left(t-\delta_{n_{1}+1}(t)\right), \cdots, x\left(t-\delta_{n}(t)\right), r(t), t\right)\right)+d M(t),
\end{aligned}
$$

where the function $\mathcal{L} U: \mathbb{R}^{d} \times \mathbb{R}^{d} \times \mathbb{S} \times \mathbb{R}_{+} \rightarrow \mathbb{R}$ is defined by

$$
\begin{aligned}
& \mathcal{L} U\left(x, y_{n_{1}+1}, \cdots, y_{n}, i, t\right)=U_{t}(x, i, t)+U_{x}(x, i, t) f(x, x \cdots, x, i, t) \\
& +\frac{1}{2} \operatorname{trace}\left[g^{T}\left(x, y_{n_{1}+1}, \cdots, y_{n}, i, t\right) U_{x x}(x, i, t) g\left(x, y_{n_{1}+1}, \cdots, y_{n}, i, t\right)\right]+\sum_{j=1}^{N} \gamma_{i j} U(x, j, t) .
\end{aligned}
$$

Moreover, the fundamental theory of calculus shows, for $j=1, \cdots, n$,

$$
\begin{aligned}
d & \left(\int _ { - \tau _ { j } } ^ { 0 } \int _ { t + s } ^ { t } \left[\tau_{j}\left|f\left(x(v), x\left(v-\delta_{1}(v)\right), \cdots, x\left(v-\delta_{n_{1}}(v)\right), r(v), v\right)\right|^{2}\right.\right. \\
& \left.\left.+\left|g\left(x(v), x\left(v-\delta_{n_{1}+1}(v)\right), \cdots, x\left(v-\delta_{n}(v)\right), r(v), v\right)\right|^{2}\right] d v d s\right) \\
= & \left(\tau _ { j } \left[\tau_{j}\left|f\left(x(t), x\left(t-\delta_{1}(v)\right), \cdots, x\left(t-\delta_{n_{1}}(v)\right), r(t), t\right)\right|^{2}\right.\right. \\
& \left.+\left|g\left(x(t), x\left(t-\delta_{n_{1}+1}(v)\right), \cdots, x\left(t-\delta_{n}(v)\right), r(t), t\right)\right|^{2}\right] \\
& -\int_{t-\tau_{j}}^{t}\left[\tau_{j}\left|f\left(x(v), x\left(v-\delta_{1}(v)\right), \cdots, x\left(v-\delta_{n_{1}}(v)\right), r(v), v\right)\right|^{2}\right. \\
& \left.\left.+\left|g\left(x(v), x\left(v-\delta_{n_{1}+1}(v)\right), \cdots, x\left(v-\delta_{n}(v)\right), r(v), v\right)\right|^{2}\right] d v\right) d t
\end{aligned}
$$


Lemma 3.1. With the notation above, $V\left(\bar{x}_{t}, \bar{r}_{t}, t\right)$ is an Itô process on $t \geq 0$ with its Itô differential

$$
d V\left(\bar{x}_{t}, \bar{r}_{t}, t\right)=L V\left(\bar{x}_{t}, \bar{r}_{t}, t\right) d t+d M(t)
$$

where $M(t)$ is a continuous local martingale with $M(0)=0$ and

$$
\begin{aligned}
L V & \left(\bar{x}_{t}, \bar{r}_{t}, t\right)=U_{x}(x(t), r(t), t)\left[f\left(x(t), x\left(t-\delta_{1}(t)\right), \cdots, x\left(t-\delta_{n_{1}}(t)\right), r(t), t\right)\right. \\
& -f(x(t), x(t), \cdots, x(t), r(t), t)] \\
& + \\
& \mathcal{L} U\left(x(t), x\left(t-\delta_{1}(t)\right), \cdots, x\left(t-\delta_{n}(t)\right), r(t), t\right) \\
& +\sum_{j=1}^{n_{1}} \theta_{j} \tau_{j}\left[\tau_{j}\left|f\left(x(t), x\left(t-\delta_{1}(t)\right), \cdots, x\left(t-\delta_{n_{1}}(t)\right), r(t), t\right)\right|^{2}\right. \\
& \left.+\left|g\left(x(t), x\left(t-\delta_{n_{1}+1}(t)\right), \cdots, x\left(t-\delta_{n}(t)\right), r(t), t\right)\right|^{2}\right] \\
& -\sum_{j=1}^{n_{1}} \theta_{j} \int_{t-\tau_{j}}^{t}\left[\tau_{j}\left|f\left(x(v), x\left(v-\delta_{1}(v)\right), \cdots, x\left(v-\delta_{n_{1}}(v)\right), r(v), v\right)\right|^{2}\right. \\
& \left.+\left|g\left(x(v), x\left(v-\delta_{n_{1}+1}(v)\right), \cdots, x\left(v-\delta_{n}(v)\right), r(v), v\right)\right|^{2}\right] d v .
\end{aligned}
$$

To study the delay-dependent asymptotic stability of the SDDE (2.1), we need to impose several new assumptions.

Assumption 3.1. Assume that there are functions $U \in C^{2,1}\left(\mathbb{R}^{d} \times \mathbb{S} \times \mathbb{R}_{+} ; \mathbb{R}_{+}\right)$, $U_{1} \in C\left(\mathbb{R}^{d} \times[-\tau, \infty) ; \mathbb{R}_{+}\right)$, and positive numbers $\alpha, \alpha_{j}(j=1, \cdots, n)$ and $\beta_{k}$ $(k=1,2,3)$ such that

$$
\sum_{j=1}^{n} \frac{\alpha_{j}}{1-\bar{\delta}_{j}}<\alpha
$$

and

$$
\begin{aligned}
& \mathcal{L} U\left(x, y_{1}, \cdots, y_{n}, i, t\right)+\beta_{1}\left|U_{x}(x, i, t)\right|^{2} \\
& \quad+\beta_{2}\left|f\left(x, y_{1}, \cdots, y_{n_{1}}, i, t\right)\right|^{2}+\beta_{3}\left|g\left(x, y_{n_{1}+1}, \cdots, y_{n}, i, t\right)\right|^{2} \\
& \leq-\alpha U_{1}(x, t)+\sum_{j=1}^{n} \alpha_{j} U_{1}\left(y_{j}, t-\delta_{j}(t)\right)
\end{aligned}
$$

for all $x, y_{1}, \cdots, y_{n} \in \mathbb{R}^{d},(i, t) \in \mathbb{S} \times \mathbb{R}_{+}$.

Assumption 3.2. Assume that there exists positive numbers $w_{j}, j=1, \cdots, n$ such that

$$
\left|f(x, x, \cdots, x, i, t)-f\left(x, y_{1}, \cdots, y_{n_{1}}, i, t\right)\right| \leq \sum_{j=1}^{n_{1}} w_{j}\left|x-y_{j}\right|
$$

for all $x, y_{1} \cdots, y_{n} \in \mathbb{R}^{d},(i, t) \in \mathbb{S} \times[-2 \tau, \infty)$.

Theorem 3.3. Let Assumptions 2.1, 2.2, 3.1 and 3.2 hold. Assume also that

$$
n_{1} \sum_{j=1}^{n_{1}} w_{j}^{2} \tau_{j}^{2} \leq 2 \beta_{1} \beta_{2} \quad \text { and } \quad n_{1} \sum_{j=1}^{n_{1}} w_{j}^{2} \tau_{j} \leq 2 \beta_{1} \beta_{3} .
$$


Then for any given initial data (2.2), the solution of the SDDE (2.1) has the properties that

$$
\int_{0}^{\infty} \mathbb{E} U_{1}(x(t), t) d t<\infty
$$

and

$$
\sup _{0 \leq t<\infty} \mathbb{E} U(x(t), r(t), t)<\infty .
$$

Proof: Fix the initial data $\eta \in C\left([-\tau, 0] ; \mathbb{R}^{d}\right)$ and $r_{0} \in \mathbb{S}$ arbitrarily. Let $k_{0}>0$ be a sufficiently large integer such that $\|\eta\|:=\sup _{-\tau \leq s \leq 0}|\eta(s)|<k_{0}$. For each integer $k>k_{0}$, define the stopping time

$$
\sigma_{k}=\inf \{t \geq 0:|x(t)| \geq k\},
$$

where throughout this paper we set $\inf \phi=\infty$ (as usual $\phi$ denotes the empty set). It is easy to see that $\sigma_{k}$ is increasing as $k \rightarrow \infty$ and $\lim _{k \rightarrow \infty} \sigma_{k}=\infty$ a.s. By the generalized Itô formula we obtain from Lemma 3.1 that

$$
\mathbb{E} V\left(\bar{x}_{t \wedge \sigma_{k}}, \bar{r}_{t \wedge \sigma_{k}}, t \wedge \sigma_{k}\right)=V\left(\bar{x}_{0}, \bar{r}_{0}, 0\right)+\mathbb{E} \int_{0}^{t \wedge \sigma_{k}} L V\left(\bar{x}_{s}, \bar{r}_{s}, s\right) d s
$$

for any $t \geq 0$ and $k \geq k_{0}$. Let $\theta_{j}=n_{1} w_{j}^{2} /\left(2 \beta_{1}\right)$. By Assumption 3.2, it is easy to see that

$$
\begin{aligned}
& U_{x}(x(t), r(t), t)\left[f\left(x(t), x\left(t-\delta_{1}(t)\right), \cdots, x\left(t-\delta_{n_{1}}(t)\right), r(t), t\right)\right. \\
& \quad-f(x(t), x(t), \cdots, x(t), r(t), t)] \\
& \leq \beta_{1}\left|U_{x}(x(t), r(t), t)\right|^{2}+n_{1} \sum_{j=1}^{n_{1}} \frac{w_{j}^{2}}{4 \beta_{1}}\left|x(t)-x\left(t-\delta_{j}(t)\right)\right|^{2} .
\end{aligned}
$$

By condition (3.4), we also have

$$
\sum_{j=1}^{n_{1}} \theta_{j} \tau_{j}^{2} \leq \beta_{2} \quad \text { and } \quad \sum_{j=1}^{n_{1}} \theta_{j} \tau_{j} \leq \beta_{3} .
$$

It then follows from Lemma 3.1 that

$$
\begin{aligned}
& L V\left(\bar{x}_{s}, \bar{r}_{s}, s\right) \leq \mathcal{L} U\left(x(s), x\left(s-\delta_{1}(s)\right), \cdots, x\left(t-\delta_{n}(s)\right), r(s), s\right)+\beta_{1}\left|U_{x}(x(s), r(s), s)\right|^{2} \\
& +\beta_{2}\left|f\left(x(s), x\left(s-\delta_{1}(s)\right), \cdots, x\left(t-\delta_{n_{1}}(s)\right), r(s), s\right)\right|^{2} \\
& +\beta_{3}\left|g\left(x(s), x\left(s-\delta_{n_{1}+1}(s)\right), \cdots, x\left(t-\delta_{n}(s)\right), r(s), s\right)\right|^{2} \\
& +n_{1} \sum_{j=1}^{n_{1}} \frac{w_{j}^{2}}{4 \beta_{1}}\left|x(s)-x\left(s-\delta_{j}(s)\right)\right|^{2} \\
& -n_{1} \sum_{j=1}^{n_{1}} \frac{w_{j}^{2}}{2 \beta_{1}} \int_{s-\tau_{j}}^{s}\left[\tau_{j}\left|f\left(x(v), x\left(v-\delta_{1}(v)\right), \cdots, x\left(v-\delta_{n_{1}}(v)\right), r(v), v\right)\right|^{2}\right. \\
& \left.+\left|g\left(x(v), x\left(v-\delta_{n_{1}+1}(v)\right), \cdots, x\left(v-\delta_{n}(v)\right), r(v), v\right)\right|^{2}\right] d v .
\end{aligned}
$$


By Assumption 3.1, we then have

$$
\begin{aligned}
& L V\left(\bar{x}_{s}, \bar{r}_{s}, s\right) \leq-\alpha U_{1}(x(s), s)+\sum_{j=1}^{n} \alpha_{j} U_{1}\left(x\left(s-\delta_{j}(s)\right), s-\delta_{j}(s)\right) \\
& \quad+n_{1} \sum_{j=1}^{n_{1}} \frac{w_{j}^{2}}{4 \beta_{1}}\left|x(s)-x\left(s-\delta_{j}(s)\right)\right|^{2} \\
& \quad-n_{1} \sum_{j=1}^{n_{1}} \frac{w_{j}^{2}}{2 \beta_{1}} \int_{s-\tau_{j}}^{s}\left[\tau_{j}\left|f\left(x(v), x\left(v-\delta_{1}(v)\right), \cdots, x\left(v-\delta_{n_{1}}(v)\right), r(v), v\right)\right|^{2}\right. \\
& \left.\quad+\left|g\left(x(v), x\left(v-\delta_{n_{1}+1}(v)\right), \cdots, x\left(v-\delta_{n}(v)\right), r(v), v\right)\right|^{2}\right] d v .
\end{aligned}
$$

Substituting this into (3.6) implies

$$
\mathbb{E} V\left(\bar{x}_{t \wedge \sigma_{k}}, \bar{r}_{t \wedge \sigma_{k}}, t \wedge \sigma_{k}\right) \leq V\left(\bar{x}_{0}, \bar{r}_{0}, 0\right)+H_{1}+\sum_{j=1}^{n_{1}}\left(H_{2}^{j}-H_{3}^{j}\right)
$$

where

$$
\begin{aligned}
H_{1} & =\mathbb{E} \int_{0}^{t \wedge \sigma_{k}}\left[-\alpha U_{1}(x(s), s)+\sum_{l=1}^{n} \alpha_{l} U_{1}\left(x\left(s-\delta_{l}(s)\right), s-\delta_{l}(s)\right)\right] d s, \\
H_{2}^{j} & =\frac{n_{1} w_{j}^{2}}{4 \beta_{1}} \mathbb{E} \int_{0}^{t \wedge \sigma_{k}}\left|x(s)-x\left(s-\delta_{j}(s)\right)\right|^{2} d s \\
H_{3}^{j} & =\frac{n_{1} w_{j}^{2}}{2 \beta_{1}} \mathbb{E} \int_{0}^{t \wedge \sigma_{k}} \int_{s-\tau_{j}}^{s}\left[\tau_{j}\left|f\left(x(v), x\left(v-\delta_{1}(v)\right), \cdots, x\left(v-\delta_{n_{1}}(v)\right), r(v), v\right)\right|^{2}\right. \\
& \left.+\left|g\left(x(v), x\left(v-\delta_{n_{1}+1}(v)\right), \cdots, x\left(v-\delta_{n}(v)\right), r(v), v\right)\right|^{2}\right] d v d s .
\end{aligned}
$$

Noting that, for $l=1, \cdots, n$,

$$
\int_{0}^{t \wedge \sigma_{k}} U_{1}\left(x\left(s-\delta_{l}(s)\right), s-\delta_{l}(s)\right) d s \leq \int_{-\delta_{l}(0)}^{t \wedge \sigma_{k}-\delta_{l}\left(t \wedge \sigma_{k}\right)} \frac{U_{1}(x(v), v)}{1-\bar{\delta}_{l}} d v \leq \int_{-\tau_{l}}^{t \wedge \sigma_{k}} \frac{U_{1}(x(v), v)}{1-\bar{\delta}_{l}} d v
$$

we have

$$
H_{1} \leq \sum_{l=1}^{n} \frac{\alpha_{l}}{1-\bar{\delta}_{l}} \int_{-\tau_{l}}^{0} U_{1}(\eta(v), v) d v-\bar{\alpha} \mathbb{E} \int_{0}^{t \wedge \sigma_{k}} U_{1}(x(s), s) d s,
$$

where $\bar{\alpha}=\alpha-\sum_{l=1}^{n} \alpha_{l} /\left(1-\bar{\delta}_{l}\right)>0$ by Assumption 3.1. Substituting this into (3.8) yields

$$
\bar{\alpha} \mathbb{E} \int_{0}^{t \wedge \sigma_{k}} U_{1}(x(s), s) d s \leq C_{1}+\sum_{j=1}^{n_{1}}\left(H_{2}^{j}-H_{3}^{j}\right),
$$

where $C_{1}$ is a constant defined by

$$
C_{1}=V\left(\bar{x}_{0}, \bar{r}_{0}, 0\right)+\sum_{l=1}^{n} \frac{\alpha_{l}}{1-\bar{\delta}_{l}} \int_{-\tau_{l}}^{0} U_{1}(\eta(s), s) d s .
$$


Applying the classical Fatou lemma and let $k \rightarrow \infty$ in (3.9) to obtain

$$
\bar{\alpha} \mathbb{E} \int_{0}^{t} U_{1}(x(s), s) d s \leq C_{1}+\sum_{j=1}^{n_{1}}\left(\bar{H}_{2}^{j}-\bar{H}_{3}^{j}\right),
$$

where

$$
\begin{aligned}
\bar{H}_{2}^{j} & =\frac{n_{1} w_{j}^{2}}{4 \beta_{1}} \mathbb{E} \int_{0}^{t}\left|x(s)-x\left(s-\delta_{j}(s)\right)\right|^{2} d s \\
\bar{H}_{3}^{j} & =\frac{n_{1} w_{j}^{2}}{2 \beta_{1}} \mathbb{E} \int_{0}^{t} \int_{s-\tau_{j}}^{s}\left[\tau_{j}\left|f\left(x(v), x\left(v-\delta_{1}(v)\right), \cdots, x\left(v-\delta_{n_{1}}(v)\right), r(v), v\right)\right|^{2}\right. \\
& \left.+\left|g\left(x(v), x\left(v-\delta_{n_{1}+1}(v)\right), \cdots, x\left(v-\delta_{n}(v)\right), r(v), v\right)\right|^{2}\right] d v d s .
\end{aligned}
$$

By the well-known Fubini theorem, we have

$$
\bar{H}_{2}^{j}=\frac{n_{1} w_{j}^{2}}{4 \beta_{1}} \int_{0}^{t} \mathbb{E}\left|x(s)-x\left(s-\delta_{j}(s)\right)\right|^{2} d s .
$$

For $t \in\left[0, \tau_{j}\right]$, we have

$$
\begin{aligned}
\bar{H}_{2}^{j} & \leq \frac{n_{1} w_{j}^{2}}{2 \beta_{1}} \int_{0}^{\tau_{j}}\left(\mathbb{E}|x(s)|^{2}+\mathbb{E}\left|x\left(s-\delta_{j}(s)\right)\right|^{2}\right) d s \\
& \leq \frac{n_{1} w_{j}^{2} \tau_{j}}{\beta_{1}}\left(\sup _{-\tau_{j} \leq v \leq \tau_{j}} \mathbb{E}|x(v)|^{2}\right) \\
& \leq \frac{n_{1} w_{j}^{2} \tau_{j}}{\beta_{1}}\left(\sup _{-\tau \leq v \leq \tau} \mathbb{E}|x(v)|^{2}\right) .
\end{aligned}
$$

For $t>\tau_{j}$, we have

$$
\bar{H}_{2}^{j} \leq \frac{n_{1} w_{j}^{2} \tau_{j}}{\beta_{1}}\left(\sup _{-\tau \leq v \leq \tau} \mathbb{E}|x(v)|^{2}\right)+\frac{n_{1} w_{j}^{2}}{4 \beta_{1}} \int_{\tau_{j}}^{t} \mathbb{E}\left|x(s)-x\left(s-\delta_{j}(s)\right)\right|^{2} d s .
$$

Noting that

$$
\begin{aligned}
& \left|x(s)-x\left(s-\delta_{j}(s)\right)\right| \\
& \leq \mid \int_{s-\tau_{j}}^{s} f\left(x(v), x\left(v-\delta_{1}(v)\right), \cdots, x\left(v-\delta_{n_{1}}(v)\right), r(v), v\right) d v \\
& \quad+\int_{s-\tau_{j}}^{s} g\left(x(v), x\left(v-\delta_{n_{1}+1}(v)\right), \cdots, x\left(v-\delta_{n}(v)\right), r(v), v\right) d B(v) \mid,
\end{aligned}
$$

we have

$$
\begin{aligned}
& \mathbb{E}\left|x(s)-x\left(s-\delta_{j}(s)\right)\right|^{2} \\
& \leq 2 \mathbb{E} \int_{s-\tau_{j}}^{s}\left[\tau_{j}\left|f\left(x(v), x\left(v-\delta_{1}(v)\right), \cdots, x\left(v-\delta_{n_{1}}(v)\right), r(v), v\right)\right|^{2}\right. \\
& \left.\quad+\left|g\left(x(v), x\left(v-\delta_{n_{1}+1}(v)\right), \cdots, x\left(v-\delta_{n}(v)\right), r(v), v\right)\right|^{2}\right] d v .
\end{aligned}
$$


Notice also that

$$
\begin{aligned}
& \int_{\tau_{j}}^{t} \mathbb{E}\left|x(s)-x\left(s-\delta_{j}(s)\right)\right|^{2} \\
& \leq 2 \mathbb{E} \int_{\tau_{j}}^{t} \int_{s-\tau_{j}}^{s}\left[\tau_{j} \mid f\left(x(v), x\left(v-\delta_{1}(v)\right), \cdots, x\left(v-\delta_{n_{1}}(v)\right), r(v),\left.v\right|^{2}\right.\right. \\
& \left.\quad+\left|g\left(x(v), x\left(v-\delta_{n_{1}+1}(v)\right), \cdots, x\left(v-\delta_{n}(v)\right), r(v), v\right)\right|^{2}\right] d v d s .
\end{aligned}
$$

Thus from (3.11) and (3.12) we get

$$
\bar{H}_{2}^{j} \leq \frac{n_{1} w_{j}^{2} \tau_{j}}{\beta_{1}}\left(\sup _{-\tau \leq v \leq \tau} \mathbb{E}|x(v)|^{2}\right)+\bar{H}_{3}^{j} .
$$

Substituting (3.13) into (3.10) yields

$$
\bar{\alpha} \mathbb{E} \int_{0}^{t} U_{1}(x(s), s) d s \leq C_{1}+2 \beta_{3} \sup _{-\tau \leq v \leq \tau} \mathbb{E}|x(v)|^{2}:=C_{2} .
$$

Letting $t \rightarrow \infty$ gives

$$
\mathbb{E} \int_{0}^{\infty} U_{1}(x(s), s) d s \leq \frac{C_{2}}{\bar{\alpha}} .
$$

Now we see from (3.8) that

$$
\mathbb{E} U\left(x\left(t \wedge \sigma_{k}\right), r\left(t \wedge \sigma_{k}\right), t \wedge \sigma_{k}\right) \leq C_{1}+\sum_{j=1}^{n_{1}}\left(H_{2}^{j}-H_{3}^{j}\right) .
$$

Letting $k \rightarrow \infty$ we get

$$
\mathbb{E} U(x(t), r(t), t) \leq C_{2}<\infty
$$

which shows

$$
\sup _{0 \leq t<\infty} \mathbb{E} U(x(t), r(t), t)<\infty .
$$

Thus the proof is complete.

Corollary 3.1. Let the conditions of Theorem 3.3 hold. If there moreover exists a pair of positive constants $c$ and $p$ such that

$$
c|x|^{p} \leq U_{1}(x, t), \quad \forall(x, t) \in \mathbb{R}^{d} \times \mathbb{R}_{+},
$$

then for any given initial data (2.2), the solution of the multiple-delay SDE (2.1) satisfies

$$
\int_{0}^{\infty} \mathbb{E}|x(t)|^{p} d t<\infty .
$$

That is, the multiple-delay SDE (2.1) is $H_{\infty}$-stable in $L^{p}$.

This corollary follows from Theorem 3.3 obviously. However, it does not follow from (3.17) that $\lim _{t \rightarrow \infty} \mathbb{E}|x(t)|^{p}=0$. 
Theorem 3.4. Let the conditions of Corollary 3.1 hold. If, moreover,

$$
p \geq 2 \quad \text { and } \quad\left(p+q_{1}-1\right) \vee\left(p+2 q_{2}-2\right) \leq q,
$$

then the solution of the multiple-delay SDE (2.1) satisfies

$$
\lim _{t \rightarrow \infty} \mathbb{E}|x(t)|^{p}=0
$$

for any initial data (2.2). That is, the variable multiple-delay SDE (2.1) is asymptotically stable in $L^{p}$.

Proof: Fix the initial data (2.2) arbitrarily. For any $0 \leq t_{1}<t_{2}<\infty$, by the Itô formula, we get

$$
\begin{array}{rl}
\mathbb{E} & \left.x\left(t_{2}\right)\right|^{p}-\mathbb{E}\left|x\left(t_{1}\right)\right|^{p} \\
& =\mathbb{E} \int_{t_{1}}^{t_{2}}\left(p|x(t)|^{p-2} x(t)^{\top} f\left(x(t), x\left(t-\delta_{1}(t)\right), \cdots, x\left(t-\delta_{n_{1}}(t)\right), r(t), t\right)\right. \\
& +\frac{p}{2}|x(t)|^{p-2}\left|g\left(x(t), x\left(t-\delta_{n_{1}+1}(t)\right), \cdots, x\left(t-\delta_{n}(t)\right), r(t), t\right)\right|^{2} \\
& +\frac{p(p-2)}{2}|x(t)|^{p-4} \mid\left(\left.x(t)^{\top} g\left(x(t), x\left(t-\delta_{n_{1}+1}(t)\right), \cdots, x\left(t-\delta_{n}(t)\right), r(t), t\right)\right|^{2}\right) d t,
\end{array}
$$

which implies

$$
\begin{aligned}
& \left.\quad|\mathbb{E}| x\left(t_{2}\right)\right|^{p}-\mathbb{E}\left|x\left(t_{1}\right)\right|^{p} \mid \\
& \leq \mathbb{E} \int_{t_{1}}^{t_{2}}\left(p|x(t)|^{p-1}\left|f\left(x(t), x\left(t-\delta_{1}(t)\right), \cdots, x\left(t-\delta_{n_{1}}(t)\right), r(t), t\right)\right|\right. \\
& \left.\quad+\frac{p(p-1)}{2}|x(t)|^{p-2}\left|g\left(x(t), x\left(t-\delta_{n_{1}+1}(t)\right), \cdots, x\left(t-\delta_{n}(t)\right), r(t), t\right)\right|^{2}\right) d t \\
& \leq \mathbb{E} \int_{t_{1}}^{t_{2}}\left(p K|x(t)|^{p-1}\left[1+|x(t)|^{q_{1}}+\sum_{j=1}^{n_{1}}\left|x\left(t-\delta_{j}(t)\right)\right|^{q_{1}}\right]\right. \\
& \left.+\frac{\left(n-n_{1}+2\right) p(p-1) K^{2}}{2}|x(t)|^{p-2}\left[1+|x(t)|^{2 q_{2}}+\sum_{j=n_{1}+1}^{n}\left|x\left(t-\delta_{j}(t)\right)\right|^{2 q_{2}}\right]\right) d t .
\end{aligned}
$$

By inequalities,

$$
\begin{aligned}
|x(t)|^{p-1}\left|x\left(t-\delta_{j}(t)\right)\right|^{q_{1}} & \leq|x(t)|^{p+q_{1}-1}+\left|x\left(t-\delta_{j}(t)\right)\right|^{p+q_{1}-1}, \\
|x(t)|^{p-1} & \leq 1+|x(t)|^{q},
\end{aligned}
$$

we can obtain

$$
\left.|\mathbb{E}| x\left(t_{2}\right)\right|^{p}-\mathbb{E}\left|x\left(t_{1}\right)\right|^{p} \mid \leq C_{3}\left(t_{2}-t_{1}\right),
$$

where

$$
\begin{aligned}
C_{3}= & p K\left(1+2\left(n_{1}+1\right) \sup _{-\tau \leq t<\infty} \mathbb{E}|x(t)|^{q}\right) \\
& +\frac{1}{2}\left(n-n_{1}+2\right) p(p-1) K^{2}\left(1+2\left(n-n_{1}+1\right) \sup _{-\tau \leq t<\infty} \mathbb{E}|x(t)|^{q}\right)<\infty .
\end{aligned}
$$

Thus we have $\mathbb{E}|x(t)|^{p}$ is uniformly continuous in $t$ on $\mathbb{R}_{+}$. By (3.17), there is a sequence $\left\{t_{l}\right\}_{l=1}^{\infty}$ in $\mathbb{R}$ such that $\mathbb{E}\left|x\left(t_{l}\right)\right|^{p} \rightarrow 0$, which easily show the claim. Thus the proof is complete. 


\section{An Example for Muptiple-delay SDEs}

Let us now discuss an example to illustrate our theory.

Example 4.1. Let us consider the SDDE with two delays (1.1), we consider two case: $\delta_{1}(t) \leq \tau=0.01$ and $\delta_{1}(t) \leq \tau=2$ for all $t \geq 0$. Let $\bar{\delta}_{1}=\bar{\delta}_{2}=0.1$ and $\delta_{2}(t)=2$ (in fact, the stability of system is independent on the size of $\delta_{2}(t)$ ). In case $\tau=0.01$, let the initial data $x(u)=2+\sin (u)$ for $u \in[-0.01,0], r(0)=2$, the sample paths of the Markovian chain and the solution of the multiple delay SDE are shown in Figure 4.1, which indicates that the multiple delay SDE is asymptotically stable. In the case $\tau=2$, let the initial data $x(u)=2+\sin (u)$ for $u \in[-2,0]$, $r(0)=2$, the sample paths of the Markovian chain and the solution of the multipledelay SDE are plotted in Figure 4.2, which indicates that the multiple-delay SDE is asymptotically unstable. From the example we can see SDDE (1.1) is stable or not depends on how long or short the time-delay is.
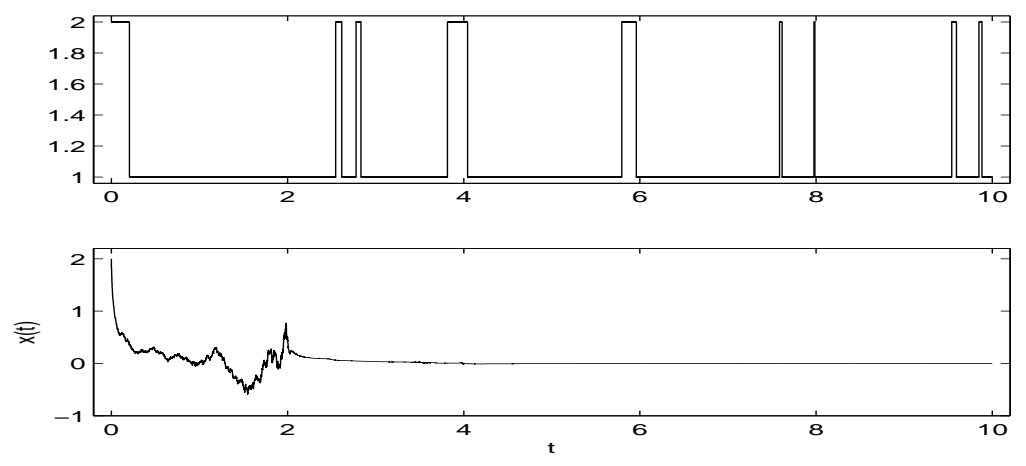

Figure 4.1: The computer simulation of the sample paths of the Markovian chain and the SDDE (1.1) with $\tau=0.01$ using the Euler-Maruyama method with step size $10^{-3}$.
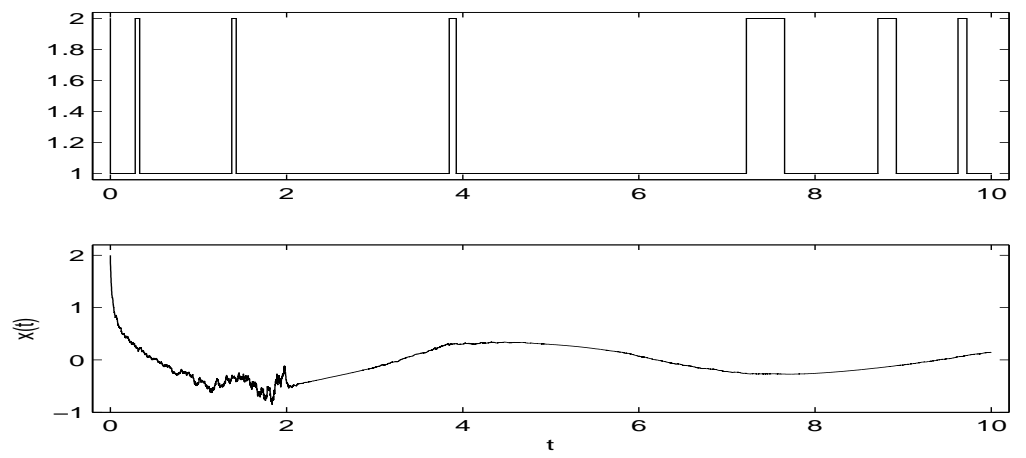

Figure 4.2 : The computer simulation of the sample paths of the Markovian chain and the SDDE (1.1) with $\tau=2$ using the Euler-Maruyama method with step size $10^{-3}$. 
We can see coefficients defined by (1.1) satisfy Assumption 2.1 with $q_{1}=3$ and $q_{2}=2$. Define $\bar{U}(x, i, t)=|x|^{6}$ for $(x, i, t) \in \mathbb{R} \times \mathbb{S} \times \mathbb{R}_{+}$. It is easy to show that

$$
\mathbb{L} \bar{U}\left(x, y_{1}, y_{2}, i, t\right)=6 x^{5} f\left(x, y_{1}, i, t\right)+15 x^{4}\left|g\left(x, y_{2}, i, t\right)\right|^{2}
$$

for $\left(x, y_{1}, y_{2}, i, t\right) \in \mathbb{R}^{3} \times \mathbb{S} \times \mathbb{R}_{+}$. We have

$$
\begin{aligned}
\mathbb{L} \bar{U}\left(x, y_{1}, y_{2}, 1, t\right)= & 6 x^{5}\left(-y_{1}-10 x^{3}\right)+\frac{15}{16} x^{4}\left(\frac{1}{4} y_{2}^{2}\right)^{2} \\
& \leq 5 x^{6}+y_{1}^{6}+\frac{15}{128} y_{2}^{6}-\left(60-\frac{15}{128}\right) x^{8}
\end{aligned}
$$

and

$$
\begin{aligned}
\mathbb{L} \bar{U}\left(x, y_{1}, y_{2}, 2, t\right)= & 6 x^{5}\left(\frac{1}{2} y_{1}-4 x^{3}\right)+\frac{15}{4} x^{4}\left(y_{2}^{2}\right)^{2} \\
& \leq 2.5 x^{6}+0.5 y_{1}^{6}-22.125 x^{8}+1.875 y_{2}^{8} .
\end{aligned}
$$

Thus, we can obtain

$$
\begin{aligned}
\mathbb{L} \bar{U}\left(x, y_{1}, y_{2}, i, t\right) & \leq 5 x^{6}+y_{1}^{6}-22.125 x^{8}+1.875 y_{2}^{8} \\
& \leq c_{1}-10\left(1+x^{8}\right)+\left(1+y_{1}^{8}\right)+2\left(1+y_{2}^{8}\right),
\end{aligned}
$$

where

$$
c_{1}=\sup _{x \in \mathbb{R}}\left\{8+5 x^{6}-12.125 x^{8}\right\}<\infty
$$

and $G(x, t)=1+x^{8}, c_{2}=10, c_{3,1}=1, c_{3,2}=2$. Therefore, Assumption 2.2 is satisfied. From Lemma 2.1, solution of the SDDE (1.1) has the that

$$
\sup _{-\tau \leq t<\infty} \mathbb{E}|x(t)|^{6}<\infty .
$$

To verify Assumption 3.1, we define

$$
U(x, i, t)=\left\{\begin{array}{cc}
x^{2}+x^{4}, & \text { if } i=1, \\
2 x^{2}+3 x^{4}, & \text { if } i=2
\end{array}\right.
$$

which shows

$$
U_{x}(x, i, t)=\left\{\begin{array}{cl}
2 x+4 x^{3}, & \text { if } i=1, \\
4 x+12 x^{3}, & \text { if } i=2
\end{array}\right.
$$

for $(x, i, t) \in \mathbb{R} \times \mathbb{S} \times \mathbb{R}_{+}$. By the equation (3.1), we have

$$
\begin{aligned}
\mathcal{L} U\left(x, y_{2}, 1, t\right)= & \left(2 x+4 x^{3}\right)\left(-x-10 x^{3}\right)+\frac{1}{32}\left(y_{2}^{2}\right)^{2}\left(2+12 x^{2}\right)-\left(x^{2}+x^{4}\right)+\left(2 x^{2}+3 x^{4}\right) \\
& \leq-x^{2}-22 x^{4}-39.875 x^{6}+\frac{1}{16} y_{2}^{4}+0.25 y_{2}^{6}
\end{aligned}
$$

and

$\mathcal{L} U\left(x, y_{2}, 2, t\right)=\left(4 x+12 x^{3}\right)\left(\frac{1}{2} x-4 x^{3}\right)+\frac{1}{8}\left(y_{2}^{2}\right)^{2}\left(4+36 x^{2}\right)+8\left(x^{2}+x^{4}\right)-8\left(2 x^{2}+3 x^{4}\right)$ 


$$
\leq-6 x^{2}-26 x^{4}-46.5 x^{6}+\frac{1}{2} y_{2}^{4}+3 y_{2}^{6} .
$$

Moreover

$$
\begin{aligned}
& \left|U_{x}(x, i, t)\right|^{2}= \begin{cases}4 x^{2}+16 x^{4}+16 x^{6}, & \text { if } i=1, \\
16 x^{2}+96 x^{4}+144 x^{6}, & \text { if } i=2 .\end{cases} \\
& \left|f\left(x, y_{1}, i, t\right)\right|^{2}=\left\{\begin{array}{l}
\left|-y_{1}-10 x^{3}\right|^{2} \leq 2 y_{1}^{2}+200 x^{6}, \text { if } i=1, \\
\left|\frac{1}{2} y_{1}-4 x^{3}\right|^{2} \leq \frac{1}{2} y_{1}^{2}+32 x^{6}, \quad \text { if } i=2 .
\end{array}\right. \\
& \left|g\left(x, y_{2}, 1, t\right)\right|^{2}=\left\{\begin{array}{l}
\frac{1}{16}\left|y_{2}^{2}\right|^{2}, \text { if } i=1, \\
\frac{1}{4}\left|y_{2}^{2}\right|^{2}, \text { if } i=2 .
\end{array}\right.
\end{aligned}
$$

Setting $\beta_{1}=0.05, \beta_{2}=0.1, \beta_{3}=4$, using (4.2)-(4.4), we obtain that

$$
\begin{aligned}
& \mathcal{L} U\left(x, y_{1}, y_{2}, i, t\right)+\beta_{1}\left|U_{x}(x, i, t)\right|^{2}+\beta_{2}\left|f\left(x, y_{1}, y_{2}, i, t\right)\right|^{2}+\beta_{3}\left|g\left(x, y_{1}, y_{2}, i, t\right)\right|^{2} \\
& \quad \leq\left\{\begin{array}{l}
-0.8 x^{2}-21.2 x^{4}-19.075 x^{6}+0.2 y_{1}^{2}+\frac{5}{16} y_{2}^{4}+\frac{1}{4} y_{2}^{6}, \quad \text { if } i=1, \\
-5.2 x^{2}-21.1 x^{4}-36.1 x^{6}+0.05 y_{1}^{2}+1.5 y_{2}^{4}+3 y_{2}^{6}, \quad \text { if } i=2 .
\end{array}\right.
\end{aligned}
$$

This implies

$$
\begin{aligned}
& \mathcal{L} U\left(x, y_{2}, i, t\right)+\beta_{1}\left|U_{x}(x, i, t)\right|^{2}+\beta_{2}\left|f\left(x, y_{1}, i, t\right)\right|^{2}+\beta_{3}\left|g\left(x, y_{2}, i, t\right)\right|^{2} \\
& \leq-0.8 x^{2}-21.1 x^{4}-19.075 x^{6}+0.2 y_{1}^{2}+1.5 y^{4}+3 y^{6} \\
& \leq-6\left(0.1 x^{2}+3 x^{4}+3 x^{6}\right)+2\left(0.1 y_{1}^{2}+3 y_{1}^{4}+3 y_{1}^{6}\right)+0.1 y_{2}^{2}+3 y_{2}^{4}+3 y_{2}^{6} .
\end{aligned}
$$

Letting $U_{1}(x, t)=0.1 x^{2}+3 x^{4}+3 x^{6}, \alpha=6, \alpha_{1}=2, \alpha_{2}=1$, we get condition (3.2). Noting that $n_{1}=1, n=2$ and $w_{1}=1$, then condition (3.4) becomes

$$
\tau \leq 0.1
$$

By Theorem 3.3, we can therefore conclude that the solution of the SDDE (1.1) has the properties that

$$
\begin{array}{r}
\int_{0}^{\infty}\left(x^{2}(t)+x^{4}(t)+x^{6}(t)\right) d t<\infty \text { a.s. and } \\
\int_{0}^{\infty} \mathbb{E}\left(x^{2}(t)+x^{4}(t)+x^{6}(t)\right) d t<\infty .
\end{array}
$$

Moreover, as $|x(t)|^{p} \leq x^{2}(t)+x^{4}(t)+x^{6}(t)$ for any $p \in[2,6]$, we have

$$
\int_{0}^{\infty} \mathbb{E}|x(t)|^{p} d t<\infty .
$$

Recalling $q_{1}=3, q_{2}=2$ and $q=6$, we see that for $p=4$, all conditions of Theorem 3.4 are satisfied and hence we have

$$
\lim _{t \rightarrow \infty} \mathbb{E}|x(t)|^{4}=0 .
$$

We perform a computer simulation with the time-delay $\tau=0.1$ for all $t \geq 0$ and the initial data $x(u)=2+\sin (u)$ for $u \in[-0.1,0]$ and $r(0)=2$. The sample paths of the Markovian chain and the solution of the SDDE (1.1) are plotted in Figure 4.3. The simulation supports our theoretical results. 

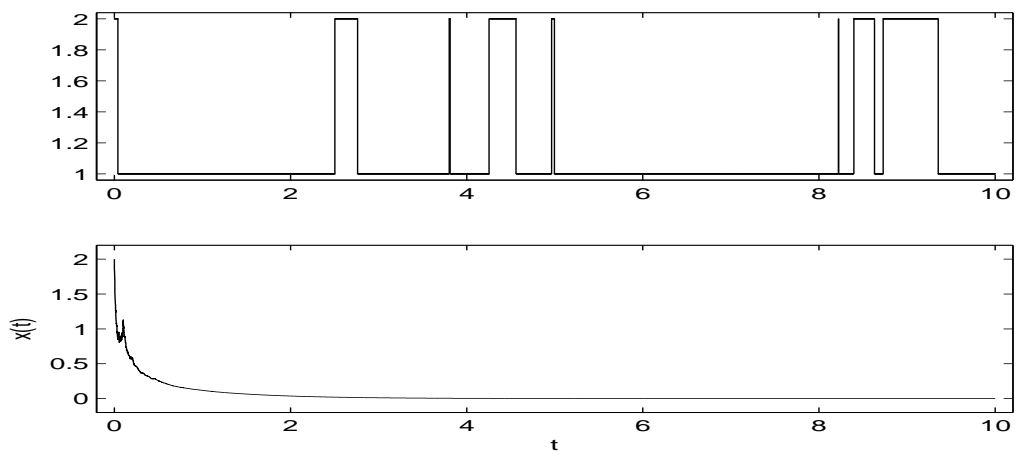

Figure 4.3 : The computer simulation of the sample paths of the Markovian chain and the SDDE (1.1) with $\tau=0.1$ using the Euler-Maruyama method with step size $10^{-3}$.

\section{Conclusion}

In real world applications, the stability and boundedness of stochastic differential delay equations are interesting topics. In this paper, we established the criteria of stability and boundedness of the solutions to SDDEs with variable multiple delays. To this end, we investigated the highly nonlinear hybrid multiple-delay SDEs. In fact, the stability of SDDEs have been studied for many years, most of the results in this topic require that the coefficients of equations are linear or nonlinear but bounded by linear functions. Recently, without the linear growth condition, Fei et al. [7] was the first to establish the delay-dependent stability criteria for highly nonlinear SDDEs by the method of Lyapunov function with a single time delay. In this paper, we obtained the results of hybrid highly nonlinear SDE with variable multiple delays. An illustrative example was given for our theory.

\section{References}

[1] A. Ahlborn and U. Parlitz, Stabilizing unstable steady states using multiple delay feedback control, Phys. Rev. Lett., 2014, 93, 264101.

[2] C. Briat, Stability analysis and stabilization of stochastic linear impulsive, switched and sampled-data systems under dwell-time constraints, Automatica, 2016, 74, 279-287.

[3] H. B. Chen, P. Shi and C. Lim, Stability analysis for neutral stochastic delay systems with Markovian switching, Syst. Control Lett., 2017, 110, 38-48.

[4] V. Dragan and H. Mukaidani, Exponential stability in mean square of a singularly perturbed linear stochastic system with state-multiplicative white-noise perturbations and Markovian switching, IET Control Theory Appl., 2016, 9, 1040-1051.

[5] C. Fei, W. Y. Fei and L. T. Yan, Existence and stability of solutions to highly nonlinear stochastic differential delay equations driven by G-Brownian motion, Appl. Math. J. Chinese Univ., to appear. 
[6] C. Fei, M. X. Shen, W. Y. Fei, X. R. Mao and L. T. Yan, Stability of highly nonlinear hybrid stochastic integro-differential delay equations, Nonlinear Anal. Hybrid Syst., 2019, 31, 180-199.

[7] W. Y. Fei, L. J. Hu, X. M. Mao and M. X. Shen, Delay dependent stability of highly nonlinear hybrid stochastic systems, Automatica, 2017, 28, 165-170.

[8] W. Y. Fei, L. J. Hu, X. R. Mao and M. X. Shen, Structured robust stability and boundedness of nonlinear hybrid delay systems, SIAM J. Control Optim., 2018, 56, 2662-2689.

[9] W. Y. Fei, L. J. Hu, X. R. Mao and M. X. Shen, Generalised criteria on delay dependent stability of highly nonlinear hybrid stochastic systems, Int. J. Robust. Nonlin., DOI: 10.1002/rnc.4402.

[10] M. Frederic, Stability analysis of time-varying neutral time-delay systems, IEEE Trans. Automat. Control, 2016, 60, 540-546.

[11] E. Fridman, Introduction to Time-Delay Systems: Analysis and Control, Birkhauser, 2014.

[12] A. Garab, V. Kovcs and T. Krisztin Global stability of a price model with multiple delays, Discrete Contin. Dyn. Syst. A., 2016,36(12),6855C6871.

[13] J. K. Hale and S. M. Lunel, Introduction to Functional Differential Equations, Springer-Verlag, 1993.

[14] L. J. Hu, X. R. Mao and Y. Shen, Stability and boundedness of nonlinear hybrid stochastic differential delay equations, Syst. Control Lett., 2013, 62, 178-187.

[15] L. J. Hu, X. R. Mao and L. G. Zhang, Robust stability and boundedness of nonlinear hybrid stochastic delay equations, IEEE Trans. Automat Control, 2013, 58(9), 2319-2332.

[16] V. B. Kolmanovskii and V. R. Nosov, Stability of Functional Differential Equations, Academic Press, London, 1986.

[17] M. L. Li and M. C. Huang, Approximate controllability of second-order impulsive stochastic differential equations with state-dependent delay, J. Appl. Anal. Comput., 2018, 8(2), 598-619.

[18] X. D. Li, Q. X. Zhu and D. O'Reganc, pth Moment exponential stability of impulsive stochastic functional differential equations and application to control problems of NNs, J. Franklin Inst., 2014, 351, 4435-4456.

[19] J. Lei and M. Mackey, Stochastic differential delay equation, moment stability, and application to hematopoitic stem cell regulation systems, SIAM J. Appl. Math., 2007, 67(2), 387-407.

[20] J. Liu, On asymptotic convergence and boundedness of stochastic systems with time-delay, Automatica, 2012, 48, 3166-3172.

[21] K. Liu, Almost sure exponential stability sensitive to small time delay of stochastic neutral functional differential equations, Appl. Math. Lett., 2018, 77, 57-63.

[22] X. R. Mao, Razumikhin-type theorems on exponential stability of stochastic functional differential eqautions, Stoch. Process. Appl., 1996, 65, 233-250.

[23] X. R. Mao, Exponential stability of stochastic delay interval systems with Markovian switching, IEEE Trans. Auto. Control, 2002, 47(10), 1604-1612. 
[24] X. R. Mao, Stochastic Differential Equations and Their Applications, 2nd Edition, Chichester: Horwood Pub., 2007.

[25] X. R. Mao, J. Lam, and L. R. Huang, Stabilisation of hybrid stochastic differential equations by delay feedback control, Syst. Control Lett., 2008, 57, 927-935.

[26] X. R. Mao and C. G. Yuan, Stochastic Differential Equations with Markovian Switching, Imperial College Press, 2006.

[27] S.-E.A. Mohammed, Stochastic Functional Differential Equations, Longman Scientific and Technical, 1984.

[28] C. Park, N. Kwon and P. Park, Optimal $H_{\infty}$ filtering for singular Markovian jump systems, Syst. Control Lett., 2018, 118, 22-28.

[29] A. Rathinasamy and M. Balachandran, Mean-square stability of semi-implicit Euler method for linear stochastic differential equations with multiple delays and Markovian switching, Appl. Math. Comput., 2008, 206, 968-979.

[30] M. X. Shen, C. Fei, W. Y. Fei and X. R. Mao, The boundedness and stability of highly nonlinear hybrid neutral stochastic systems with multiple delays, Sci. China Inf. Sci., revised.

[31] M. X. Shen, W. Y. Fei, X. R. Mao and S. N. Deng, Exponential stability of highly nonlinear neutral pantograph stochastic differential equations, Asian J. Control, DOI: 10.1002/asjc.1903.

[32] M. X. Shen, W. Y. Fei, X. R. Mao and Y. Liang, Stability of highly nonlinear neutral stochastic differential delay equations, Syst. Control Lett., 2018, 115, $1-8$.

[33] S. Y. Xu, J. Lam and X. R. Mao, Delay-dependent $H_{\infty}$ control and filtering for uncertain Markovian jump systems with time-varying delays, IEEE Trans. Circuits Syst. I, 2007, 54(9), 2070-2077.

[34] S. R. You, W. Liu, J. Q. Lu, X. R. Mao and J. W. Qiu, Stablization of hybrid systems by feedback control based on discrete-time state observation, SIAM J. Contrl Optim., 2015, 53(2), 905-925.

[35] D. Yue and Q. Han, Delay-dependent exponential stability of stochastic systems with time-varying delay, nonlinearity, and Markovian switching, IEEE Trans. Automat. Control, 2005, 50, 217-222.

[36] Q. X. Zhu, Stability analysis of stochastic delay differential equations with Lévy noise, Syst. Control Lett., 2018, 118, 62-68. 